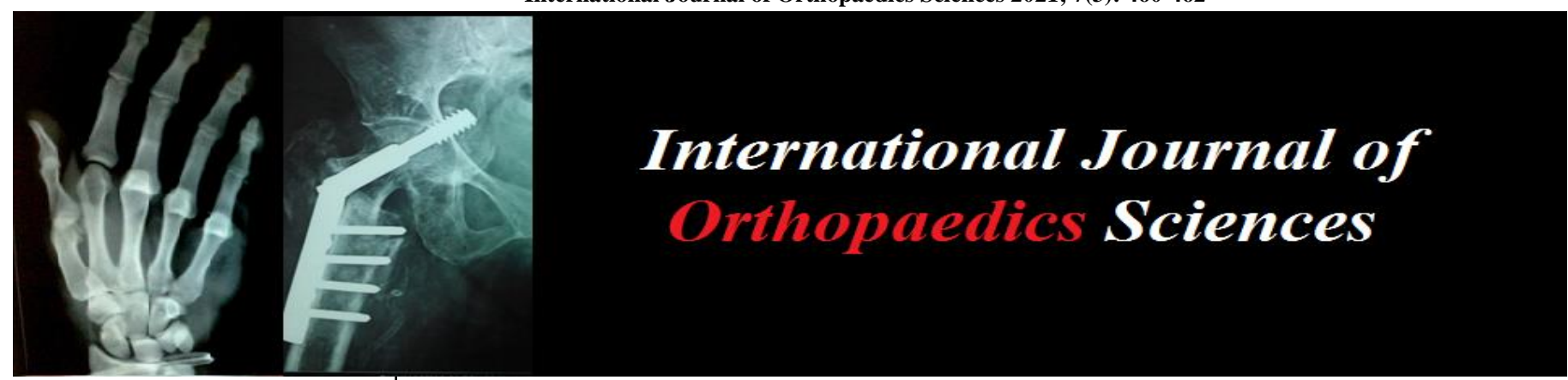

E-ISSN: 2395-1958

P-ISSN: 2706-6630

IJOS 2021; 7(3): 460-462

(C) 2021 IJOS

www.orthopaper.com

Received: 03-05-2021

Accepted: 07-06-2021

\section{Dhaval Gotecha}

MS Orthopedics, Govt. Doon

Medical College, Dehradun,

Uttarakhand, India

\section{Akshat Mittal}

DNB Orthopedics. Govt. Doon

Medical College, Dehradun,

Uttarakhand, India

\section{Effect of platelet-rich plasma in the treatment of patellar tendinopathy}

\section{Dhaval Gotecha and Akshat Mittal}

DOI: $\underline{\text { https://doi.org/10.22271/ortho.2021.v7.i3g.2788 }}$

\section{Abstract}

Aim: The aim of this study was to evaluate the therapeutic effect of platelet-rich plasma (PRP) injections in the treatment of Patellar Tendinopathy.

Methods: The study group comprised of 39 patients comprising mainly athletes with patellar tendinopathy for more than 6 months. PRP was obtained from the patients' own blood and injected in a single dose. Peppering technique was used for injecting the doses in the patients. Results were calculated using pre-injection and post-injection Visual Analog Scale (VAS) and Victorian Institute of Sport Assessment (Patellar, VISA-P) at baseline, 6 weeks and 6 months.

Results: All patients enrolled in the study completed a 6 month follow-up. There were no complaints of any side-effects to the administered platelet-rich plasma. No infection or any other complications were reported at the end of 3 months. The outcome was seen in terms of VAS and VISA-P.

Conclusion: PRP therapy proves to be effective in relieving pain in a long term for treatment of Patellar Tendinopathy.

Keywords: knee pain, athletes, platelet-rich plasma, Tibial tuberosity, patellar tendon

\section{Introduction}

Patellar tendinopathy (PT), a degenerative disease of the patellar tendon, results in anterior knee pain associated with focal and palpable tenderness at the inferior pole of the patella ${ }^{[1,2]}$. Magnetic resonance imaging (MRI) show smaller tendon cross-sectional area ${ }^{[3]}$ and abnormalities of the posterior border of the patellar tendon and infrapatellar fat pad ${ }^{[4]}$ like tendon thickening, increased blood flow, and signal intensity changes, although these findings may also occur among asymptomatic athletes ${ }^{[5]}$.

The activities that require jumping, running, or rapid changes in direction ${ }^{[6,7]}$ often result in patellar tendinopathy. More prevalent in sporting activities that involve jumping, also known as "jumper's knee." ${ }^{[8]}$ and has a prevalence ranging from $14 \%$ to $32 \%$ and $45 \%$ in basketball and volleyball athletes, respectively ${ }^{[7]}$.

Chronic repetitive tendon overload is the most commonly proposed theory ${ }^{[9,10]}$. Overload may result in weakening of the tissue and failure ${ }^{[10]}$. The increased strain is located in the deep posterior portion of the tendon, closer to the center of rotation of the knee and the inferior pole of the patella, especially with increased knee flexion ${ }^{[9]}$. Microscopic failure occurs within the tendon at high loads and eventually leads to alterations at the cellular level, which weaken the mechanical properties ${ }^{[10]}$. Tendon microtrauma can lead to individual fibril degeneration due to stress across the tendon, which can accumulate over time, leading to chronic tendinopathy [10].

A large number of treatment options have been considered in treating Patellar Tendinopathy including Non steroidal anti-inflammatory drugs (NSAIDs), physiotherapy, and ultrasonic therapy, Rest, stretching exercises. Corticosteroid injections locally have also been used over the past few years. Over the recent few years platelet-rich plasma (PRP) injections have also been used with promising results.

The purpose of this study was to evaluate the therapeutic effect of PRP injections in the treatment of Patellar Tendinopathy.

\section{Corresponding}

DNB Orthopedics. Govt. Doon

Medical College, Dehradun,

Uttarakhand, India 


\section{Materials and Methods}

This retrospective study includes 39 patients diagnosed with chronic Patellar Tendinopathy between July 2017 and July 2019. Total patients were 46 out of which 7 patients lost follow up. The diagnosis was done through MRI showing increased T2 signal intensity at the Inferior pole of patella and clinically by the same Orthopaedic team as characteristic knee pain lasting for more than a period of 6 months, localized along the Inferior pole of Patella. All the patients had symptoms non-responsive or recurrence of symptoms following conservative and physical therapy.

Patients with previous history of fracture or surgery on the affected knee, those with previous history of steroid injections, infections or systemic diseases, arthritis, radiculopathy, and patients on anti-platelet medication and oral steroids were excluded from the study. All patients were instructed to stop taking NSAIDs

3 weeks before the procedure.

\section{Procedure}

$30 \mathrm{ml}$ of patients' blood was withdrawn and inserted into prepacked PRP kits (Tricell) along with $5 \mathrm{ml}$ of anticoagulant $10 \%$ sodium citrate. The PRP sample was prepared by a double centrifugation process. The first centrifuge was done at $3200 \mathrm{rpm}$ for $4 \mathrm{~min}$. The cellular component was separated from the fluid component and a second centrifuge was them performed at $3300 \mathrm{rpm}$ for $3 \mathrm{~min}$. Following this, approximately 3-4 $\mathrm{ml}$ was obtained. The maximally tender spot on the Inferior pole of patella, then injected $3 \mathrm{~mL}$ of Local Anaesthesia with adrenaline combination subcutaneously using sterile technique. Care was taken not to anesthetize the tendon or tendon sheath. The injection was done using the peppering technique, where multiple punctures were done on the Patellar Tendon. The study was explained to every patient and informed consent was obtained from them before the procedure.

After the procedure, Ice was applied for pain as desired by the patient. For 48 hours after injection, patients were instructed to refrain from exercise. After 1 week, rehabilitation began. All patients were advised Full weight bearing and gradual return to activities after 1 week of the procedure.

Pre-procedure and on final follow-up, patients were assessed for their symptoms using visual analog scale (VAS) and Victorian Institute of Sport Assessment (Patellar, VISA-P).

\section{Results}

The present study included 39 patients. The mean age of patients was 21.3 years, comprising a total of 31 males $(79.5 \%)$ and 8 females $(20.5 \%)$. the right knee was affected in 28 patients $(71.8 \%)$ whereas the left knee was affected in 10 patients $(25.6 \%)$ and bilateral knees were affected in 1 patient $(2.56 \%)$.

None of the patients included in the study had complaints of any side effects after administering PRP. No infection or any other complications were reported at the end of 6 months. The mean VAS score at baseline, at 6 weeks follow-up and at 6 months follow-up were $8.3 \pm 1,1.5 \pm 0.8$ and $0.8 \pm 0.8$. The mean VISA-P score at baseline, at 6 weeks follow-up and at 6 months follow-up were $33.6 \pm 8.6,61.2 \pm 4.5$ and $86.9 \pm 2.4$. The differences in the pre-treatment scores and follow up scores were statistically significant. All patients returned to daily routine activites of preinjury state.

\section{Discussion}

The present study aimed to compare the efficacy of PRP in the treatment of Patellar Tendinopathy. In our study, we found significant differences relative to VAS and VISA-P scores before treatment, 6 weeks after treatment and 6 months after treatment.

In our study, PRP was administered at the point of maximum tenderness and MRI findings of the knee at inferior pole of patella. Our results are generally consistent with other reports supporting the efficacy of PRP in the treatment of chronic patellar tendinopathy.

The results of our study suggested that PRP was associated with significant improvement in VAS and VISA-P scores. Our results were consistent with other studies in literature. Since PRP obtained from autologous blood, there is no risk of immune reaction or disease transfer. There are no studies in the literature warning of hyperplasia, carcinogenesis or tumor growth of PRP ${ }^{[11,12]}$.

It is possible that the volume of the PRP injection plays a role in its biological effects. This volume, regardless of the injected contents, may improve tendinopathies via mechanical disruption of neo neuro vascularization. For example, Crisp et al. ${ }^{[13]}$ found that $22-$ to $50-\mathrm{mL}$ combination injections of bupivacaine, hydrocortisone, and normal saline generated significant improvements in VAS and

VISA scores among patellar tendinopathy patients. Vetrano study and others have included 2 or more PRP injections ${ }^{[14-}$ 16]. Whereas ours involved only a single PRP injection.

In our study, No complications were encountered in any patient. Muto et al. ${ }^{[17]}$ performed a study on the effect of PRP and corticosteroids on human rotator-cuff derived cells. In their study, they showed that while PRP and corticosteroids both show a progressive decrease in inflammatory markers on the target tissue, corticosteroids have shown to have an increase in degenerative markers in contrast to PRP which shows a decrease in the degenerative markers on the target tissue.

Our study had a few limitations. First, we did not have a control group. Peppering technique was used to administer PRP to the target tissue; hence, the response obtained could be attributed to the technique itself. We did not document anatomic tendon changes by ultrasound or MRI after treatment. With the use of PRP, we did not measure the precentrifuge and post-centrifuge platelet concentration in any of the samples; hence, no standard dose of administration could be quantified.

\section{Conclusion}

In conclusion, although limited by a few factors, the present study suggested that PRP was as effective as other treatments in terms of pain and functional results in the treatment of patients with Patellar Tendinopathy.

\section{References}

1. Blazina ME, Kerlan RK, Jobe FW, Carter VS, Carlson GJ. Jumper's knee. Orthop Clin North Am 1973;4(3):665-678.

2. Warden SJ, Brukner P. Patellar tendinopathy. Clin Sports Med 2003;22(4):743-759.

3. Couppe C, Kongsgaard M, Aagaard P et al. Differences in tendon properties in elite badminton players with or without patellar tendinopathy. Scand J Med Sci Sports 2013;23(2):e89-95.

4. Warden SJ, Kiss ZS, Malara FA, Ooi AB, Cook JL, Crossley KM. Comparative accuracy of magnetic resonance imaging and ultrasonography in confirming clinically diagnosed patellar tendinopathy. Am J Sports 
Med. 2007;35(3):427-436.

5. Cook JL, Ptazsnik R, Kiss ZS, et al. High reproducibility of patellar tendon vascularity assessed by colour Doppler ultrasonography: A reliable measurement tool for quantifying tendon pathology. $\mathrm{Br} \mathrm{J}$ Sports Med. 2005;39(10):700-703.

6. Ferretti A. Epidemiology of jumper's knee. Sports Med. 1986; 3(4):289-295.

7. Lian OB, Engebretsen L, Bahr R. Prevalence of jumper's knee among elite athletes from different sports: a crosssectional study. Am J Sports Med. 2005;33(4):561-567.

8. Lian OB, Engebretsen L, Bahr R: Prevalence of jumper's knee among elite athletes from different sports: A crosssectional study. Am J Sports Med 2005;33(4):561-567.

9. Kannus P, Jozsa L, Natri A. Effects of training, immobilization and remobilization on tendons. Scand J Med Sci Sports 1997;7:67-71.

10. Rees JD, Maffulli N, Cook J. Management of tendinopathy. Am J Sports Med 2009;37:1855-1867.

11. Anitua E, Andia I, Ardanza B, Nurden P, Nurden AT. $\mathrm{Au}$-dolorous platelets as a source of proteins for healing and tissue regeneration Romb Haemost 2004;91:4-15.

12. Sanchez AR, Sheridan PJ, Kupp LI. Is platelet-rich plasma the perfect enhancement factor? A current review, Int J Oral Maxillofac Implants 2003;18:93-103.

13. Crisp $\mathrm{T}$, Khan $\mathrm{F}$, Padhiar $\mathrm{N}$ et al. High volume ultrasound guided injections at the interface between the patellar tendon and Hoffa's body are effective in chronic patellar tendinopathy: A pilot study. Disabil Rehabil 2008;30(20-22):1625-1634.

14. Kon E, Filardo G, Delcogliano M et al. Platelet-rich plasma: new clinical application: A pilot study for treatment of jumper's knee. Injury 2009;40(6):598-603.

15. Vetrano M, Castorina A, Vulpiani MC, Baldini R, Pavan A, Ferretti A. Platelet-rich plasma versus focused shock waves in the treatment of jumper's knee in athletes. Am J Sports Med 2013;41(4):795-803.

16. Filardo G, Kon E, Della Villa S, Vincentelli F, Fornasari PM, Marcacci M. Use of platelet-rich plasma for the treatment of refractory jumper's knee. Int Orthop 2010;34(6):909-915.

17. Muto T, Kokubu T, Mifune Y et al. Effects of plateletrich plasma and triamcinolone acetonide on interleukin$1 \beta$-stimulated human rotator cuff-derived cells Bone Joint Res 2016;5(12):602-609. 